\title{
A Novel Range-free Localization Algorithm for Anisotropic Networks
}

\author{
Jaehun Lee, Wooyong Chung and Euntai Kim* \\ School of Electrical and Electronic Engineering, Yonsei University, Seoul, Korea
}

\begin{abstract}
In this paper, a novel range-free localization algorithm for anisotropic networks is proposed. In the proposed method, the characteristics of the given network are considered and each sensor node estimates the relation between the hop counts and the geographical distances. Unlike most of the previous localization algorithms, the proposed method performs well not only in the isotropic network but also in the anisotropic networks. The proposed method is applied to both isotropic and anisotropic network topologies and the simulation results demonstrate that the method exhibits excellent and robust performances.
\end{abstract}

Keywords: Wireless sensor networks, Localization, Range-free algorithm.

\section{Introduction}

Recently, wireless sensor networks (WSNs) have been used in many applications including target tracking [1], environmental monitoring [2], search and rescue [3], etc. In general, WSN consists of many sensor nodes which have small memories and communicate with other nodes within the given communication range. In many WSN applications, the position information of each sensor node is very useful but not given. The localization, or location estimation, of sensor nodes is one of the main issues in WSN.

There are many research conducting on localization problem in WSNs [4-10] and they can be divided into two groups, range-based algorithms and range-free algorithms. The rangebased algorithms estimate the location of unknown sensor nodes based on measurements by additional hardware. The range-based algorithms might provide quite accurate localization results, but they need some additional hardware for distance or angle measurements. Increasing the estimation accuracy of those measurements is also another problem of the range-based algorithms. The range-free algorithms use only the connectivity information among sensor node [5]. The rangefree algorithms are cheaper and simpler than the range-based methods since they do not need any additional hardware and therefore might be more appropriate to the large-scale WSNs which consist of hundreds or thousands of sensor nodes. The main problem of the range-free localization algorithms is improving the localization accuracy to the suitable level.

In this paper, a novel range-free localization algorithm is proposed. This work is motivated by DV-hop method [6] in

Manuscript received Sep. 2, 2011; revised Nov. 4, 2011; accepted Nov. 7;

${ }^{+}$Corresponding author

This work was supported by "Development of Commercialization Element Technology for Market-friendly Intelligent Transport Robot" (Project number: 20110324) of the Ministry of Knowledge Economy, Republic of Korea. which the average hop distance was computed and applied to the sensor nodes for localization. To estimate the unknown locations of sensor nodes, the distances between sensor node and anchor nodes, whose positions are known a priori, are needed. Therefore, the method of transforming hop count to distance is essential to the range-free localization algorithms. For this reason the average hop distance is calculated and used in [6]. DV-hop method works well in case of isotropic network since the sensor and anchor nodes are placed in the entire area uniformly. However, it results in large errors in case of anisotropic networks since the nodes are not uniformly distributed and the relationship between hop counts and geographic distances is very weak.

The proposed method resolves the problem of DV-hop by reflecting the characteristics of the given WSN on estimation of the average hop distances. In the proposed method, each sensor node calculates its own average hop distances between itself and all anchor nodes considering the sensor distribution of the given sensor network. Since the proposed method considers the characteristics of the given network, it shows much better performances in case of anisotropic network especially.

The rest of this paper is organized as follows: In Section 2, detailed explanations about DV-hop localization algorithm which is the motivation of this paper are given. In Section 3, the proposed method is presented. In Section 4, some simulations are conducted and the results of the proposed method are compared with those of previous methods. Finally, a conclusion is drawn in Section 5.

\section{DV-hop Localization Algorithm}

DV-hop method [6] is one of the most famous range-free localization algorithms. It works well in case of isotropic network since the sensor and anchor nodes are placed in the entire area uniformly. However, it results in large errors in case of anisotropic networks since the nodes are not uniformly distributed and the relationship between hop counts and geographic distances is very weak. The details of DV-hop 
algorithm is presented as follows.

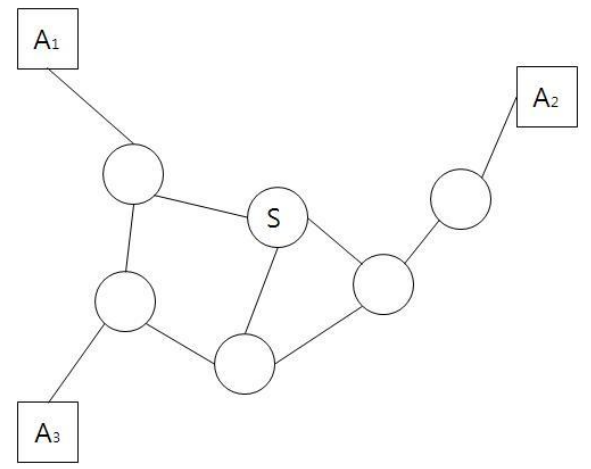

Fig. 1. An example of the given WSN.

Considering the given WSN in Fig. 1, nodes $A_{1}, A_{2}$, and $A_{3}$ are anchor nodes whose locations are known a priori and node $S$ is the sensor node whose location should be estimated. It is assumed that each anchor node knows the hop counts and distances between anchor nodes that are shown in Table 1 .

Table 1. Hop counts and distances between anchor nodes

\begin{tabular}{|c|c|c|c|}
\hline & $A_{1}$ and $A_{2}$ & $A_{2}$ and $A_{3}$ & $A_{3}$ and $A_{1}$ \\
\hline Hop count & 5 & 5 & 3 \\
\hline Distance & $120 \mathrm{~m}$ & $150 \mathrm{~m}$ & $100 \mathrm{~m}$ \\
\hline
\end{tabular}

Then, each anchor node $A_{i}$ calculates its average hop distance, which is also called as a correction, $c_{i}$ based on the following equation

$$
c_{i}=\frac{\sum_{j} \sqrt{\left(x_{i}-x_{j}\right)^{2}+\left(y_{i}-y_{j}\right)^{2}}}{\sum_{j} h_{i, j}}
$$

where $x_{i}$ and $y_{i}$ represent the coordinates of the anchor node $A_{i}$ and $h_{i, j}$ is the hop count between anchor node $A_{i}$ and $A_{j}$. After calculating the average hop distance, each anchor node broadcasts it to all sensor nodes. Then, each sensor node estimates the distances between itself and anchor nodes based on the received average hop distances. Finally, each sensor node estimates its position by multilateration [10]. Table 2 shows the average hop distances and estimated distances between sensor node $S$ and anchor nodes $A_{1}, A_{2}$, and $A_{3}$.

Table 2. Avg. hop distances and estimated distances from $S$

\begin{tabular}{|c|c|c|c|}
\hline & node $A_{1}$ & node $A_{2}$ & node $A_{3}$ \\
\hline $\begin{array}{c}\text { Avg. hop } \\
\text { distance }\end{array}$ & $c_{1}=\frac{120+100}{5+3}$ & $c_{2}=\frac{120+150}{5+5}$ & $c_{3}=\frac{100+150}{3+5}$ \\
\hline $\begin{array}{c}\text { Est. } \\
\text { distance }\end{array}$ & $27.5 \times 2=55$ & $27 \times 3=81$ & $31.25 \times 3=93.75$ \\
\hline
\end{tabular}

\section{Modified DV-hop Localization Algorithm with Constraints}

In this section, a novel range-free localization algorithm is proposed. The proposed method is the extended version of [11] which modifies the DV-hop algorithm using the shortest path information. The main idea of the method in [11] is that each sensor node calculates its own average hop distances to all anchor nodes.

Consider a given WSN $S N=\left\{S_{1}, \cdots, S_{M}, A_{1}, \cdots, A_{N}\right\}$ consists of $M$ sensor nodes $\left\{S_{1}, \cdots, S_{M}\right\}$ and $N$ anchor nodes $\left\{A_{1}, \cdots, A_{N}\right\}$, where $M \square N$. The location of each sensor and anchor nodes is defined as

$$
\begin{aligned}
& \mathbf{L}\left(A_{i}\right)=\left(x_{i}, y_{i}\right)^{T}, \\
& \mathbf{L}\left(S_{i}\right)=\left(\bar{x}_{i}, \bar{y}_{i}\right)^{T} .
\end{aligned}
$$

Then, each sensor node $S_{i}$ calculates its own average hop distance vector $\mathbf{c}_{i}=\left[\begin{array}{llll}c_{i, 1} & c_{i, 2} & \cdots & c_{i, N}\end{array}\right]$ based on the following equation

$$
c_{i, j}=\sum_{k}\left(\frac{\sqrt{\left(x_{j}-x_{k}\right)^{2}+\left(y_{j}-y_{k}\right)^{2}}}{h_{j, k}}\right),
$$

$$
k=\underset{k}{\arg \min }\left|h_{j, i}+h_{i, k}-h_{j, k}\right| \text { subject to }\left\{\begin{array}{c}
k \in\{1,2, \cdots, M\} \\
k \neq i
\end{array},\right.
$$

where $h_{j, k}$ is the hop count between anchor node $A_{j}$ and $A_{k}$. More formally, the $k$ th anchor node $A_{k}$ is used in calculating the average hop distance between the $j$ th anchor node $A_{j}$ and the sensor node $S_{i}$ if the shortest path between $A_{j}$ and $A_{k}$ includes the sensor node $S_{i}$. For example, in case of the WSN in Fig. 2, the sensor node $S_{1}$ is not on the shortest way between $A_{1}$ and $A_{3}$ but on the way between $A_{1}$ and $A_{2}$. Therefore, the anchor node $A_{3}$ is not considered when $c_{1,1}$, the average hop distance between $S_{1}$ and $A_{1}$, is calculated. On the other hand, the sensor node $S_{2}$ is on the both shortest way between $A_{1}$ and $A_{3}$ and between $A_{1}$ and $A_{2}$. Therefore, both anchor nodes $A_{2}$ and $A_{3}$ are considered when $c_{2,1}$, the average hop distance between $S_{2}$ and $A_{1}$, is calculated. Table 3 shows the average hop distances and estimated distances.

Table 3. Average hop distances and estimated distances

\begin{tabular}{|c|c|c|c|}
\hline & node $A_{1}$ & node $A_{2}$ & node $A_{3}$ \\
\hline $\begin{array}{c}\text { Avg. hop } \\
\text { distance for } \\
S_{1}\left(=\mathbf{c}_{1}\right)\end{array}$ & $c_{1,1}=\frac{120}{5}=24$ & $c_{1,2}=\frac{120}{5}=24$ & $c_{1,3}=\frac{150}{5}=30$ \\
\hline $\begin{array}{c}\text { Est. distance } \\
\text { for } S_{1}\end{array}$ & $24 \times 2=48$ & $24 \times 3=72$ & $30 \times 3=90$ \\
\hline $\begin{array}{c}\text { Avg. hop } \\
\text { distance for } \\
S_{2}\left(=\mathbf{c}_{2}\right)\end{array}$ & $c_{2,1}=\frac{120+100}{5+3}$ & $c_{2,2}=\frac{120}{5}=24$ & $c_{2,3}=\frac{100}{3}=33.3$ \\
\hline $\begin{array}{c}\text { Est. distance } \\
\text { for } S_{2}\end{array}$ & $27.5 \times 1=27.5$ & $24 \times 4=96$ & $33.3 \times 2=66.6$ \\
\hline
\end{tabular}




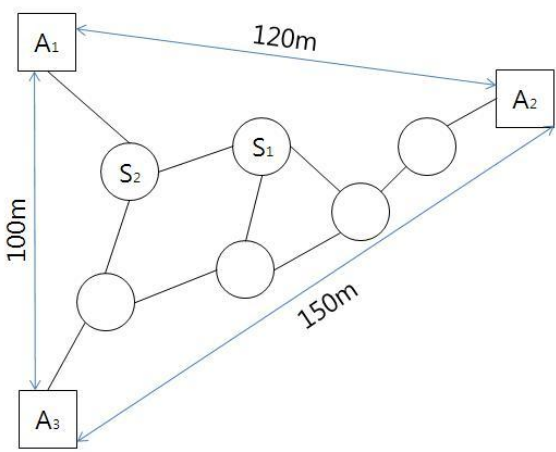

Fig. 2. An example of WSN for modified DV-hop algorithm.

The drawbacks of this method are that it only considers whether the sensor node is on the shortest way between two anchors. In case of anisotropic network, some anchor nodes that are very close to each other can have large hop count. In this case, the average hop count information is rare useful. For example, let us consider the sensor network in Fig. 3.

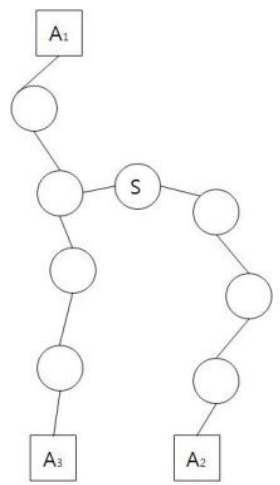

Fig. 3. An example of the anisotropic network.

Suppose that the sensor node $S$ calculates average hop distance to the anchor node $A_{3}$. The sensor node $S$ is on the way of the shortest path between $A_{3}$ and $A_{2}$, but it is not on the way between $A_{3}$ and $A_{1}$. However, it is more accurate if we consider the anchor node $A_{1}$ when calculating the average hop distance from sensor node $S$ to the anchor node $A_{3}$. To resolve this problem, the proposed method considers the physical distances and hop counts between anchor nodes when calculating the average hop distance vector. The following theorem is the constraints that we can use in the proposed method.

\section{Theorem 1}

Suppose that all nodes in a sensor network $S$ are in a straight line. If the geographical distance $d_{i, j}$ between two nodes $s_{i}$ and $s_{j}$ is smaller than $k$ times and longer than $k-1$ times of communication range $R$ ( $\left.(k-1) R<d_{i, j}<k R\right)$, then the maximum hop count between two nodes $s_{i}$ and $s_{j}$ is $2 k-1$.

Proof. Assume that the hop count between two nodes $s_{i}$ and $s_{j}$ is larger than $2 k-1$. Then, there are at least $2 k-1$ nodes $\left\{\hat{s}_{1}, \hat{s}_{2}, \cdots, \hat{s}_{2 k-1}\right\}$ between $s_{i}$ and $s_{j}$ with following conditions,

$$
\begin{aligned}
& \hat{d}_{l, l+1}<R \\
& \hat{d}_{m, m+2}>R \\
& \left\|s_{i}-\hat{s}_{1}\right\|<R \\
& \left\|s_{i}-\hat{s}_{2}\right\|>R \\
& \left\|s_{j}-\hat{s}_{2 k-1}\right\|<R \\
& \left\|s_{j}-\hat{s}_{2 k-2}\right\|>R
\end{aligned}
$$

where $\hat{d}_{i, j}$ is the distance between $\hat{s}_{i}$ and $\hat{s}_{j}$, $l=1, \cdots, 2 k-2$ and $m=1, \cdots, 2 k-3$. Consider a straight line between two nodes $s_{i}$ and $s_{j}$ is segmented to $k$ segments and each segment is not larger than $R$. The first segment includes the node $s_{i}$ and the last segment, the $k$ th segment, includes the node $s_{j}$ as shown in Fig. 4. It is clear that the first and last segments can include one node $\hat{s}_{1}$ and $\hat{s}_{2 k-1}$, respectively, because of the above condition (4-2) and (4-3). The number of rest segments is $k-2$ and the number of rest nodes that should be included in the segments is $2 k-3$. Therefore, at least one segment should include 3 nodes and it is in contradiction to the above condition (4-1) since the length of each segment is smaller than $R$. Therefore, the assumption at the beginning, i.e. the hop counts between two nodes $s_{i}$ and $s_{j}$ is larger than $2 k-1$, is false and the maximum hop counts between two nodes $s_{i}$ and $s_{j}$ is $2 k-1$.

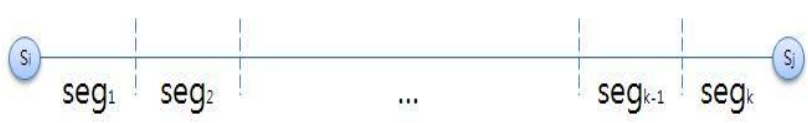

Fig. 4. Segmentation of the given straight line.

In the proposed method, each sensor node considers the physical conditions between anchor nodes based on the above theorem. By the above theorem, we can ignore the anchor nodes that are very close to each other can have large hop count when we calculate the average hop distances. Finally, each sensor node $S_{i}$ calculates its own average hop distance vector

$$
\begin{aligned}
& \mathbf{c}_{i}=\left[\begin{array}{llll}
c_{i, 1} & c_{i, 2} & \cdots & c_{i, N}
\end{array}\right] \text { with the following equation } \\
& c_{i, j}=\sum_{k}\left(\frac{\sqrt{\left(x_{j}-x_{k}\right)^{2}+\left(y_{j}-y_{k}\right)^{2}}}{h_{j, k}}\right) \\
& k=\underset{k}{\arg \min }\left|h_{j, i}+h_{i, k}-h_{j, k}\right| \\
& \text { s.t. } k \in\{1,2, \cdots, M\} \\
& k \neq i \\
& 2\left\lceil\frac{d_{j, k}}{R}\right\rceil-1 \geq h_{j, k}
\end{aligned}
$$


where \lceil\rceil is the ceiling function which gives the smallest integer greater than or equal to the argument. Each sensor node estimates its own location based on multilateration of its distances to all anchor nodes in the distributed manner. Several multilateration algorithms have been reported for localization in the WSN and a non-iterative multilateration algorithm [10] is used in this paper.

\section{Experimental Results}

In this section, we have conducted some simulations to see the performance of proposed method compared with the previous method: DV-hop [6]. In all simulations, two kinds of networks are considered: an isotropic network and anisotropic network. In the isotropic network, all sensor and anchor nodes are distributed uniformly in the given field. The main characteristics of isotropic network are that the connectivity of all directions in any points of the given field resembles. In the anisotropic network, on the other hand, sensor and anchor nodes are placed randomly. The distribution of the nodes may be different according to the directions at some positions because of obstacles such as walls or other reasons. Fig. 5 and 6 shows the isotropic and anisotropic network used in this paper, respectively.

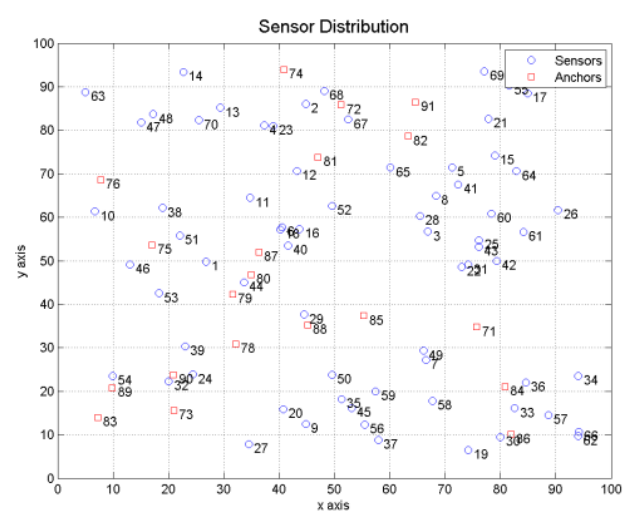

Fig. 5. Isotropic sensor network topology.

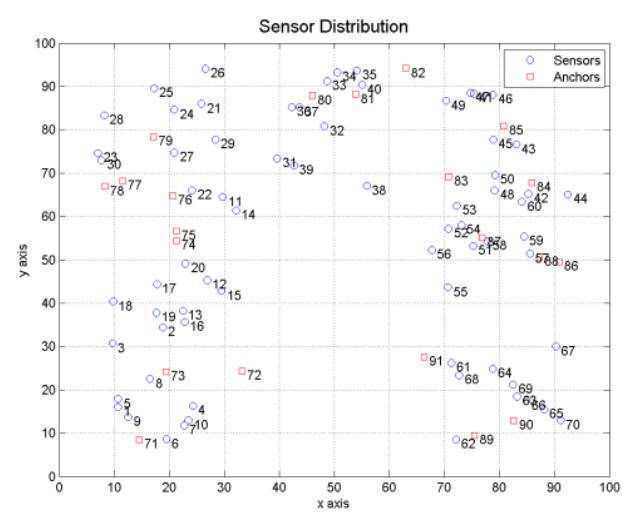

Fig. 6. Anisotropic sensor network topology.
The communication range $R$ for all nodes is set to 15 with respect to the $100 \times 100$ square region and 10 independent simulation runs are made for each topology. The total size of the network is fixed to 300 and the anchor ratio is changed from $10 \%$ to $30 \%$. Localization performances are compared for isotropic and anisotropic networks in Figs. 7 and 8, respectively.

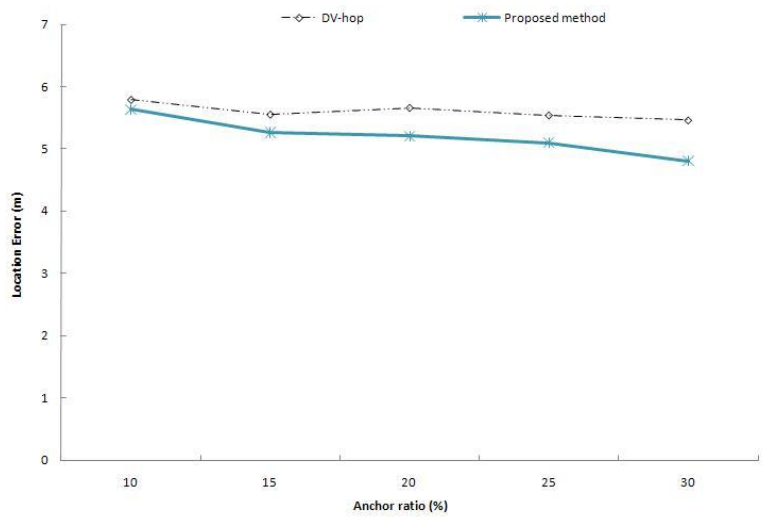

Fig. 7. Comparison of the average position error for the isotropic topology under various anchor populations.

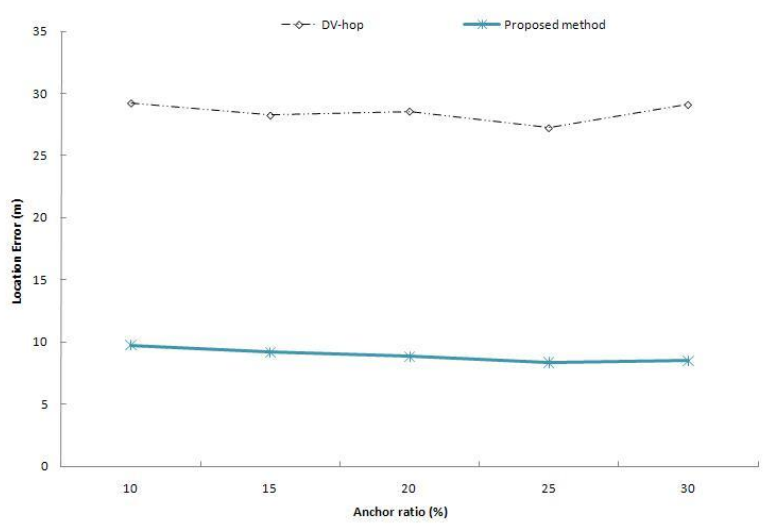

Fig. 8. Comparison of the average position error for the anisotropic topology under various anchor populations.

It can be seen that the proposed method outperforms the previous methods in both isotropic and anisotropic networks. Especially, the proposed method shows much more superior to DV-hop method in case of anisotropic networks. The reason might be that DV-hop algorithm does not consider the characteristics of the given network but the proposed method considers not only the topology of the given network but also the relations between physical distances and hop counts.

Next, more realistic environment is concerned. We take into consideration an irregular radio propagation model which is used in [12]. In this model, the communication range is varied by the direction because of the various physical reasons including the multipath channel. The irregularity of the model is represented by DOI (degree of irregularity) and the actual 
communication range of each node is represented by

$$
(1-D O I) R \leq \text { actual range } \leq R
$$

Fig 9 shows the example of the radio propagation model.

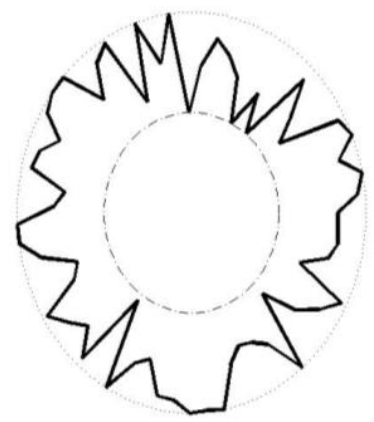

Fig. 9. Radio propagation model for the realistic environment.

The total network size is fixed to 300 and the anchor ratio is varied from $10 \%$ to $30 \%$. The DOI value is as assumed to be 0.2 and the maximum communication range $R$ is set to be 10. The localization performances for isotropic and anisotropic networks are compared in Figs. 10 and 11, respectively.

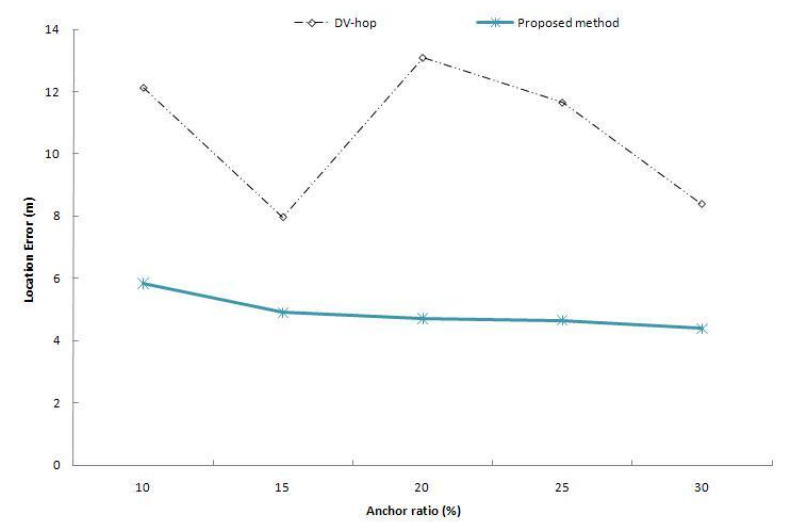

Fig. 10. Comparison of the average position error for the isotropic topology under noisy radio propagation.

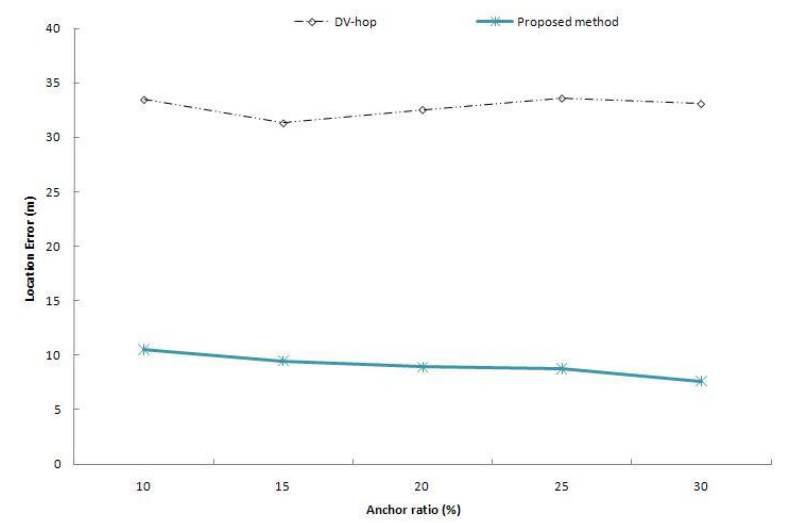

Fig. 11. Comparison of the average position error for the anisotropic topology under noisy radio propagation.
As in the previous simulation, the proposed method outperforms the previous DV-hop method on average and demonstrates more consistent performance. It can be seen that the proposed method is rarely influenced by the radio propagation model which means that it is more robust to the various real environments.

\section{Conclusions}

In this paper, we have proposed a novel range-free algorithm for localization in wireless sensor networks. The proposed method considers both the characteristics of the given network and the relation between the geographical distance and the connection of the network. As a result, the proposed method works well not only in the isotropic network but also in the anisotropic network. The simulations are conducted to see the performance of the proposed method. We have considered two communication models: 1) ideal case and 2) fluctuating model for both isotropic and anisotropic networks. The simulation results demonstrated superior performance of the proposed method compared with previous method.

\section{References}

[1] S. Lee, H. Cha, and R. Ha, "Energy-aware location error handling for object tracking applications in wireless sensor networks," Computer Communications, vol. 30, no. 7, pp. 1443-1450, 2007.

[2] A. Mainwaring, J. Polastre, R. Szewczyk, and D. Culler, "Wireless sensor networks for habitat monitoring," in Proc. ACM Workshop on Sensor Networks and Applications, pp. 88-97, 2002.

[3] G. M. Hoffmann and C. J. Tomlin, "Mobile sensor network control using mutual information methods and particle filters," IEEE Transactions on Automatic Control, vol. 55, no. 1, pp. 32-47, 2010.

[4] G. Mao, B. Fidan, and B. D. O. Anderson, "Wireless sensor network localization techniques," Computer Networks, vol. 51, no. 10, pp. 2529-2553, 2007.

[5] Y. Shang, W. Ruml, Y. Zhang, and M. Fromherz, "Localization from connectivity in sensor networks," IEEE Transactions on Parallel and Distributed Systems, vol. 15, no. 11, pp. 961-974, 2004.

[6] D. Niculescu and B. Nath, "DV based positioning in ad hoc networks," Journal of Telecommunication Systems, vol. 22, pp. 267-280, 2003.

[7] S. Yun, J. Lee, W. Chung, and E. Kim, "Localization method in wireless sensor networks using fuzzy modeling and genetic algorithm," Journal of Korean institute of intelligent systems, vol. 18, no. 4, pp. 530-536, 2008.

[8] K. Yedavalli and B. Krishnamachari, "Sequence-based localization in wireless sensor networks," IEEE Transactions on Mobile Computing, vol. 7, no. 1, pp. 81-94, 
2008.

[9] S. Yun, J. Lee, W. Chung, E. Kim, and S. Kim, "A soft computing approach to localization in wireless sensor networks," Expert Systems with Applications, vol. 36, no. 4, pp. 7552-7561, 2009.

[10] K. Langendoen and N. Reijers, "Distributed localization in wireless sensor networks: a quantitative comparison," Computer Networks, vol. 43, no. 4, pp. 499-518, 2003.

[11] J. Lee, W. Chung, and E. Kim, "Efficient range-free localization algorithm for wireless sensor network based on shortest path information," in Proc. of ICROS-SICE International Joint Conference 2009, pp. 1962-1965, 2009.

[12] J. P. Sheu, P. C. Chen, and C. S. Hsu, "A distributed localization scheme for wireless sensor networks with improved grid-scan and vector-based refinement," IEEE Transactions on Mobile Computing, Vol. 7, No. 9, pp. 1110-1123, 2008.

\section{Jaehun Lee}

Ph.D. candidate of the Yonsei University

Research Area: Computational Intelligence, Localization and Tracking in Sensor Network.

E-mail : aznable17@yonsei.ac.kr

\section{Wooyong Chung}

$\mathrm{Ph}$.D. candidate of the Yonsei University

Research Area: Fuzzy Control, Artificial Intelligence, Localization and Tracking in Sensor Network.

E-mail : wychung@yonsei.ac.kr

\section{Euntai Kim}

Professor of the Yonsei University

Research Area: Computational Intelligence, Intelligent Vehicular Technology, Mobile Robot.

E-mail : etkim@yonsei.ac.kr 